\title{
Joint Interference Management in Ultra-Dense Small Cell Networks: A Multi-Domain Coordination Perspective
}

\author{
Jia Xiao*, Chungang Yang*, Alagan Anpalagan ${ }^{\dagger}$, Qiang $\mathrm{Ni}^{\ddagger}$, Mohsen Guizani ${ }^{\S}$
}

\begin{abstract}
Extensive deployment of heterogeneous small cells in cellular networks results in ultra-dense small cell networks (USNs). USNs have been established as one of the vital networking architectures in the 5G to expand system capacity and augment network coverage. However, intensive deployment of cells results in a complex interference problem. In this paper, we propose a distributed multi-domain interference management scheme among cooperative small cells. The proposed scheme mitigates the interference while optimizing the overall network utility. Additionally, we jointly investigate OFDMA scheduling, TDMA scheduling, interference alignment (IA), and power control. We model small cells' coordination behavior as an overlapping coalition formation game (OCFG). In this game, each base station can make an autonomous decision and participate in more than one coalition to perform IA and suppress intra-coalition interference. To achieve this goal, we propose a distributed joint interference management (JIM) algorithm. The proposed algorithm allows each small cell base station to self-organize and interact into a stable overlapping coalition structure and reduce interference gradually from multi-domain, thus achieving an optimal tradeoff between costs and benefits. Compared with existing approaches, the proposed JIM algorithm provides appreciable performance improvement in terms of total throughput, which is demonstrated by simulation results.
\end{abstract}

Index Terms-Ultra-dense small cell networks, coalition game theory, OFDMA scheduling, interference alignment, TDMA scheduling.

\footnotetext{
* J. Xiao and C. Yang are with the State Key Laboratory on Integrated Services Networks, Xidian University, Xi'an, 710071 China (emails: \{xiao_jia2008, chgyang2010\}@163.com).

I A. Anpalagan is with the Department of Electrical and Computer Engineering, Ryerson University, Toronto, ON, Canada (email: alagan@ee.ryerson.ca).

$\ddagger$ Q. Ni is with School of Computing and Communications, Lancaster University, Lancaster, UK. (email: q.ni@lancaster.ac.uk).

3 M. Guizani is with Electrical and Computer Engineering Department University of Idaho, Idaho, USA. (email: mguizani@ieee.org).

Part of this work has been presented at IEEE 8th International Conference on Wireless Communications and Signal Processing (WCSP 2016).

This work was supported in part by the Natural Science Basic Research Plan in Shaanxi Province of China (2017JZ021); by a special financial grant from the China Postdoctoral Science Foundation (2016T90894); by a special financial grant from the Shaaxi Postdoctoral Science Foundation (154066); by the CETC Key Laboratory of Data Link Technology (CLDL-20162309); by the ISN02080001 and ISN90106180001; by the 111 Project under Grant B08038; and by the Shaanxi Province Science and Technology Research and Development Program (2011KJXX-40); This work was supported in part by the National Science Foundation of China $(61671062,91638202)$; This work was also supported by the Spanish MINECO through the project TEC201785587-R of the National Research and Innovation Program (partially financed with FEDER funds). This work is supported in part by the EPSRC IAA project CSA7113, EPSRC project EP/K011693/1 and EU CROWN project PIRSESGA-2013-610524.
}

\section{INTRODUCTION}

Ultra-dense small cell networks (USNs) are emerging as one of the most promising solutions to tackle the 1000-fold capacity increase in the next generation wireless communication systems [1]. The basic idea of USNs is to increase network densification by deploying the various heterogeneous small cells. Generally speaking, small cell base stations (SBSs) are plug-and-play, low-power, low-cost, and can only support short-range data services [2]. USNs have the potential to broad the coverage and increase the network throughput with improved spectrum utilization [3].

However, the intensive deployment of various types of small cells causes a severe co-tier interference problem in high population density areas resulting in a significant reduction of the operational efficiency of USNs and affecting the performance of whole network. Therefore, interference management becomes an important issue to resolve. Centralized interference management techniques involve heavy signaling overhead [4], which limits their applications in a large density of small cells. The work [5] proposed a joint load balance and interference mitigation scheme, which was a centralized scheme. The macrocell needed to collect and operate so much information that the scheme was not suitable for dense networks. In [6], a semi-clustering of victim-cells paradigm for co-tier interference mitigation has been proposed in USNs, but sacrificing the performance of the non-critical users. A usercentric QoS-aware interference coordination scheme has been proposed in [7] where its complexity increased sharply with the increase of base station density. Thus, it is important to design a suitable and low-complexity distributed scheme to reduce the interference further. In this respect, distributed interference management has been studied extensively, e.g., power control [8], [9], fractional frequency reuse [10], [11], resource allocation [12], [13], hybrid user access policies [14], [15], interference coordination [16], [17], interference alignment (IA) [18], [19]. These techniques mitigate the interference in different dimensions, e.g., time, frequency, spatial, and power.

Many existing works attempted to manage interference in the frequency domain, e.g., fractional frequency reuse (FFR) [10], [11], OFDMA scheduling [23], [24]. In [10], the fractional frequency reuse has been adapted to allocate orthogonal bandwidth to reduce the interference for femtocells. In [11], the authors conducted a broad comparison among four different FFR schemes, namely soft FFR, FFR-3, strict FFR, and OSFFR in terms of the total throughput in two-tier 
heterogeneous networks. In [23], self-configuration and selfoptimization techniques have been used to guide the OFDMA spectrum allocation to mitigate the interference. Authors in [24] proposed an adaptive graph coloring approach for resource allocation, whose goal was to achieve fairness among users and manage interference among femtocells. Due to the scarcity of spectrum resource, only considering frequency domain interference management was inefficient, especially in USNs.

TDMA scheduling is a typical method of interference management in the time domain [25], [26]. A randomized distributed TDMA scheduling (RD-TDMA) algorithm to generate a feasible schedule and handle the correlated contention quickly has been presented in [25]. In [26], the authors formulated the cooperation of SBSs as an overlapping coalition formation game (OCFG) to manage the interference, where each coalition avoided the co-tier interference by the use of TDMA scheduling. Although TDMA scheduling can exploit the time-varying wireless channels, the finite time-slot limits the further deployment of interference management.

IA is a powerful interference management scheme in the space domain [28], [29]. The successively determined beamforming matrices for macro base stations (MBSs) and SBSs have been presented in a hierarchical interference alignment scheme [28]. Moreover, the above works provided an additional degree of freedom compared to the conventional interference coordination schemes which use a time domain based resource partitioning. In [29], a cooperative spectrum leasing scheme has been presented for primary and secondary users to balance the tension between revenue collection/payment and data transmission, where the data transmission is coordinated by IA. Meanwhile, the solution of IA is subjected to a particular dimension and cooperative costs, which hinders the direct deployment of IA.

Power optimization has been regarded as an important approach to the interference management problem in the power domain [30], [31]. In [30], a novel geometrical method has been proposed to obtain the capacity region and the optimal input power spectral densities by single-user water-filling argument. In [31], a non-cooperative game has been presented to optimize power allocation. The existence and uniqueness of Nash equilibrium of the game has been checked in [31]. The power optimization can weaken the co-tier interference. However, a simple power optimization is not satisfactory for managing interference in USNs.

The interference problem becomes severe with the increase in network densification, making it difficult to solve by considering only one dimension. Therefore, in order to optimize the overall network throughput, it is desirable to perform interference management by implementing advanced physical layer techniques for different domains jointly.

Most existing literature focused on non-cooperation interference management schemes, where each SBS behaves selfishly and irrationally. In such a non-cooperative environment, each SBS is only concerned about its own performance, while ignoring the damage it causes to other SBSs. With the development of advanced cooperative networking paradigms, SBSs can form clusters to cooperatively coordinate their transmis- sions with fundamental limits and sustainable costs [20]. In [19], a distributed cooperation mechanism has been proposed for femtocells to form a proper cluster, where the advanced interference alignment techniques have been performed in each cluster. In [21], a distributed algorithm for spectrum sharing among the femtocell has been proposed based on a transferable utility cooperative game. In [22], authors proposed a distributed cooperative energy efficiency maximization game algorithm to maximize the system energy efficiency by optimizing the spectrum efficiency of SBSs, mainly maximized from spectrum and power domain. Nevertheless, the formed coalitions are not allowed to overlap in the mentioned works, i.e., each SBS can only participate in not more than one coalition. This restriction tremendously limits SBSs to achieve higher gains from the cooperation. Thus, the concept of OCFG has been adopted to achieve a better performance in [26], [27].

Several literature attempted to address the interference problem from multiple domain perspective. For instance, in [29], a joint power control and IA scheme has been proposed from the spectral and space domains perspectives to enhance the utility of users. The work [32] proposed a coordinated scheme including the decoupling of scheduling, beamforming, and power allocation steps. However, the complexity of the scheme increased sharply with the number of base stations. Distinguished from the existing literature, in this paper, we propose a distributed joint interference alignment (JIM) algorithm to solve the co-tier interference problem from the multi-domain perspective, including frequency, time, space, and power domain. By using the proposed JIM algorithm, each SBS can reduce the interference from neighboring SBSs by OFDMA scheduling in the frequency domain first. Next, the SBSs form the overlapping coalitions based on the history set and transfer rule, where the intra-coalition interference is aligned by IA in the space domain. Each overlapping coalition satisfies the IA feasibility conditions. Then, the inter-coalition interference is mitigated by TDMA scheduling in the time domain. Finally, we apply power optimization to achieve better performance. Meanwhile, we provide stability of the proposed overlapping coalitional structure. Simulation results demonstrate that the proposed JIM scheme significantly improves performance gains compared to other classical methods. In comparison with [33], we divide the original problem into multiple subproblems, and give corresponding steps in the algorithm table. Besides, we analyze the complexity of the algorithm. The main contributions of this paper are summarized as follows:

- Multi-Domain Interference Management: We jointly investigate the interference management from multidomain perspective to mitigate the co-tier interference in USNs, where four advanced physical layer techniques including the OFDMA scheduling, IA, TDMA scheduling, and power optimization are implemented.

- Cooperation Framework and Cost: We formulate SBSs' cooperation behavior as an OCFG to improve the cooperation gains, where the related cooperation cost is taken into account.

- Decentralized Problem Formulation: We formulate the 
joint multi-domain interference management problem as a decentralized problem. The primal problem is an NPhard problem which is hard to solve directly. Thus we decompose this original problem into four subproblems which are solved sequentially.

- Distributed JIM Algorithm: We present a distributed JIM algorithm, where SBSs can reduce, avoid, and manage complex interference from multiple domains.

The remainder of this paper is organized as follows. In section II, we describe the system model and formulate the joint multi-domain interference management problem. In section III, we introduce some relevant concepts of OCFG and propose a distributed JIM algorithm. In section IV, the practical deployment and application are introduced. Simulation results are presented and analyzed in section $\mathrm{V}$, and finally, conclusions are drawn in section VI.

Notation 1: In the remainder of the paper, $\log$ refers to the base-2 logarithm. Bold uppercase letters (e.g., $\boldsymbol{A}$ ) denote matrices, bold lowercase letters (e.g., $\boldsymbol{a}$ ) denote column vectors, and normal letters (e.g., $a$ ) denote scalars. $\operatorname{rank}(\boldsymbol{A})$ represents the singular value of a matrix $\boldsymbol{A} \cdot \mathrm{v}_{\mathrm{d}}(\boldsymbol{A})$ represents $d$-th smallest eigenvalue's corresponding eigenvector. $\mathbb{C}^{N \times M}$ denotes the set of complex $N \times M$ matrices and $(.)^{\dagger}$ represents the Hermitian transpose operator.

\section{System Model and Problem Formulation}

Before formulating the joint multi-domain interference management problem, we first introduce the system model and illustrate the distributed IA.

\section{A. System Model}

We consider a downlink transmission of an OFDMA heterogeneous USN, e.g., shopping mall, metro station, and openair assembly, which consists of an MBS and $N$ SBSs, as illustrated in Fig. 1. Let the terms $\mathcal{N}=\{1, \ldots, N\}$ and $\mathcal{L}_{n}=\left\{1, \ldots, L_{n}\right\}$ denote the set of all SBSs and it serves a set of small cell user equipments (SUEs). The access method of MBS and all SBSs is the closed access. In the network, SBSs are connected to each other via a wired backhaul. Each SBS $n \in \mathcal{N}$ chooses a sub-channel set $\mathcal{K}_{n}$ as the initial frequency resource, which contains $\left|\mathcal{K}_{n}\right|=K$ orthogonal frequency sub-channels from a total set of sub-channels $\mathcal{K}$ in a frequency division duplexing access mode. Each SUE $l \in \mathcal{L}_{n}$ is separately scheduled on orthogonal sub-channel $k \in \mathcal{K}_{n}$. Each transmitter-receiver pair operates over a wireless channel during a time-slot, which is separately equipped with $N_{t}$ transmit antennas and $N_{r}$ receive antennas. The set of available time-slots is $T$ during a scheduling period in TDMA mode. The interference problem in USNs with a traditional non-cooperative scenario is depicted in Fig. 1 . The set \{SUE 1, SUE 2, SUE 7\} reuses the same sub-channel 1 during the same time-slot. Thus they separately receive the interference signal from the SBS of the set. The same situations occur in sets $\{$ SUE 3, SUE 4, SUE 6\}, \{SUE 5, SUE 8$\}$ and $\{$ SUE 9, SUE $10\}$. It is obvious that the co-tier interference will be more severe with the increase of SBSs's density. In order to solve the joint multi-domain interference management problem, two

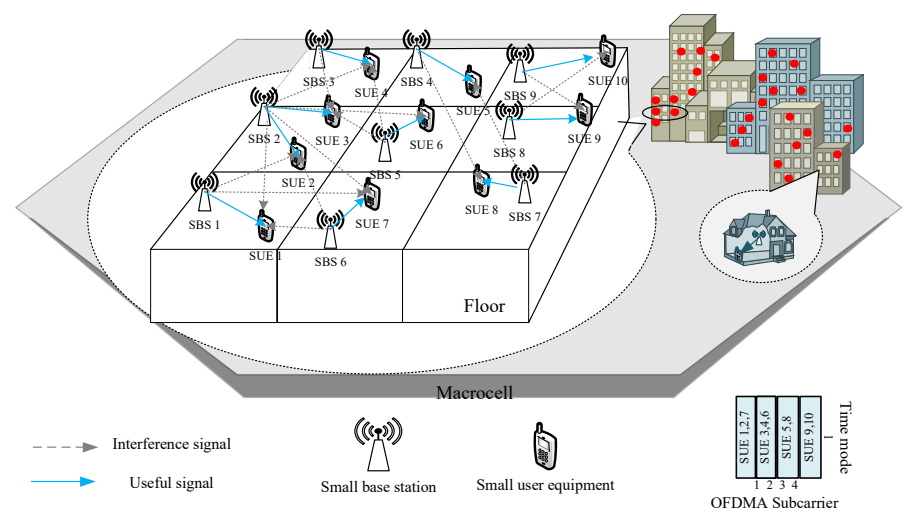

Fig. 1. An illustrative example of heterogeneous ultra-dense small cell networks.

fundamental questions need to be answered: firstly, what is the order of different domains in the joint optimization of physical layer technologies to solve the co-tier interference problem; secondly, how to motivate the SBSs to adopt the joint interference management scheme.

Given the transmission from an SBS $n$ to one of its SUE $l \in \mathcal{L}_{n}$ on sub-channel $k \in \mathcal{K}_{n}$ in a non-cooperative way, the received signal $\mathbf{y}_{n l}^{k, t}$ for SUE $l$ at a given time-slot $t \in T$ is:

$$
\boldsymbol{y}_{n l}^{k, t}=\alpha_{n l}^{k} \beta_{n l}^{t} \boldsymbol{H}_{n l}^{k} \boldsymbol{V}_{n}^{k} \boldsymbol{x}_{n}^{k}+\sum_{i \in \mathcal{N}, i \neq n} \alpha_{i l^{\prime}}^{k} \beta_{i l^{\prime}}^{t} \boldsymbol{H}_{i l}^{k} \boldsymbol{V}_{i}^{k} \boldsymbol{x}_{i}^{k}+\mathbf{n}_{l},
$$

where the term $\boldsymbol{H}_{n l}^{k} \in \mathbb{C}^{N_{r} \times N_{t}}$ denotes the MIMO channel matrix between SUE $l$ and SBS $n$ on sub-channel $k$. Moreover, it is assumed that all elements of each channel matrix $\boldsymbol{H}_{n l}^{k}$ are independent random variables. The term $\boldsymbol{V}_{n}^{k} \in \mathbb{C}^{N_{t} \times d_{n}}$ denotes the associated encoding matrix on the sub-channel $k$. In addition, the term $d_{n}$ denotes the degree of freedom of the transmitter-receiver pair (i.e., the multiplexing gain). The term $\boldsymbol{x}_{n}^{k} \in \mathbb{C}^{d_{n} \times 1}$ denotes the $d_{n}$-dimensional data signal vector from SBS $n$ on sub-channel $k$. The term $\alpha_{n l}^{k}$ denotes the binary element of the local subcarrier allocation matrix, i.e., $\boldsymbol{\alpha}=\left[\alpha_{n l}^{k}\right] \in\{0,1\} . \alpha_{n l}^{k}=1$ indicates the SBS $n$ transmits the signal to SUE $l$ over sub-channel $k$, otherwise $\alpha_{n l}^{k}=0$. Similarly, the term $\beta_{n l}^{t}$ denotes the binary element of TDMA time-slot allocation matrix, i.e., $\boldsymbol{\beta}=\left[\beta_{n l}^{k}\right] \in\{0,1\}$. $\beta_{n l}^{t}=$ 1 indicates the transmission from SBS $n$ to SUE $l$ is active during the time-slot $t \in T$, and otherwise $\beta_{n l}^{t}=0$. Moreover, the term $\boldsymbol{V}_{i}^{k} \in \mathbb{C}^{N_{t} \times d_{i}}$ and the term $\boldsymbol{x}_{i}^{k} \in \mathbb{C}^{d_{i} \times 1}$ are the associated encoding matrix, and the $d_{i}$-dimensional data signal to SBS $i$ on sub-channel $k$, the term $\boldsymbol{n}_{l} \in \mathbb{C}^{N_{r} \times 1}$ denotes zero mean unit variance circularly symmetric additive white gaussian noise (AWGN) vector at receiver $l$. The term $l^{\prime} \in \mathcal{L}_{i}$ is the SUE served by SBS $i$ on sub-channel $k$.

\section{B. Distributed IA}

The basic principle of IA is to align the interference from undesirable transmitters in no more than half of received signal dimensions. The dimensions of received signal are a geometrical subspace which can be defined by a subset of the available antennas at the receivers [34]. This enables the transmitter to achieve a higher degree of freedom with interferencefree dimensions. Meanwhile, the complete knowledge of the 
channel state information is required at each participating transmitters to align the interference perfectly. To meet this challenge, an algorithm has been provided to compute the interference cancellation matrix $\boldsymbol{U}_{l}^{k} \in \mathbb{C}^{N_{r} \times d_{n}}$ and the precoding matrix $\boldsymbol{V}_{i}^{k}$ in [35]. Only local channel state information at each interfering transmitters is needed to achieve IA by utilizing the reciprocity of wireless channels in the iterative distributed IA algorithm. In addition, the general IA feasibility conditions can be summarized according to [34], [36]:

$$
\left(\boldsymbol{U}_{l}^{k}\right)^{\dagger} \boldsymbol{H}_{i l}^{k} \boldsymbol{V}_{i}^{k}=0, \forall n \in S, \forall i \in S \backslash n, \forall l \in \mathcal{L}_{n},
$$

and

$$
\operatorname{rank}\left(\left(\boldsymbol{U}_{l}^{k}\right)^{\dagger} \boldsymbol{H}_{n l}^{k} \boldsymbol{V}_{n}^{k}\right)=d_{n}>0, \forall n \in S, \forall l \in \mathcal{L}_{n} .
$$

Here, the term $S$ denotes a coalition (or a cluster) of SBSs, where the interference from members of the same coalition is aligned in a special signal dimension. Equation (2) implies that the interference from the SBSs $i \in S \backslash n$ will be aligned along a vector which is orthogonal to the desired signal vector. The achieved degree of freedom is represented in (3). Based on (2) and (3), the existence of a solution for IA has been constrained by the dimensions of $\left(|S|, N_{t}, N_{r}\right)$ as discussed in [36], where the term $|S|$ represented the size of the coalition. For example, IA is available under the symbol extension is equal to one and (4) is satisfied, the constraint can be expressed as follow:

$$
d \times(|S|+1) \leq N_{t}+N_{r} .
$$

where $d$ denotes the degree of freedom, i.e., the multiplexing gain. For the sake of the discussion, the target multiplexing gain at each receiver is fixed. For example, the existence of a solution for IA is simplified to $N_{t}+N_{r} \geq|S|+1$ under the target multiplexing gain is equal to one.

Hence, when the size of coalition satisfies (4), the interference from SBSs of the same coalition can be aligned by IA and obtain the estimated signal $\boldsymbol{y}_{n l}^{k, t}$ at a given time-slot $t$ as in (5):

$$
\begin{aligned}
\left(\boldsymbol{U}_{l}^{k}\right)^{\dagger} \boldsymbol{y}_{n l}^{k, t}= & \alpha_{n l}^{k} \beta_{n l}^{t}\left(\boldsymbol{U}_{l}^{k}\right)^{\dagger} \boldsymbol{H}_{n l}^{k} \boldsymbol{V}_{n}^{k} \boldsymbol{x}_{n}^{k} \\
& +\underbrace{\sum_{i \in S, i \neq n} \alpha_{i l^{\prime}}^{k} \beta_{n l^{\prime}}^{t}\left(\boldsymbol{U}_{l}^{k}\right)^{\dagger} \boldsymbol{H}_{i l}^{k} \boldsymbol{V}_{i}^{k} \boldsymbol{x}_{i}^{k}}_{0} \\
& +\sum_{j \in \mathcal{N} \backslash S} \alpha_{j l^{\prime \prime}}^{k} \beta_{j l^{\prime \prime}}^{t}\left(\boldsymbol{U}_{l}^{k}\right)^{\dagger} \boldsymbol{H}_{j l}^{k} \boldsymbol{V}_{j}^{k} \boldsymbol{x}_{j}^{k}+\boldsymbol{U}_{l}^{k} \boldsymbol{n}_{l} . \\
\Rightarrow \tilde{\boldsymbol{y}}_{n l}^{k, t}= & \alpha_{n l}^{k} \beta_{n l}^{t}\left(\boldsymbol{U}_{l}^{k}\right)^{\dagger} \boldsymbol{H}_{n, l}^{k} \boldsymbol{V}_{n}^{k} \boldsymbol{x}_{n}^{k} \\
& +\sum_{j \in \mathcal{N} \backslash S} \alpha_{j l^{\prime \prime}}^{k} \beta_{j l^{\prime \prime}}^{t}\left(\boldsymbol{U}_{l}^{k}\right)^{\dagger} \boldsymbol{H}_{j l}^{k} \boldsymbol{V}_{j}^{k} \boldsymbol{x}_{j}^{k}+\left(\boldsymbol{U}_{l}^{k}\right)^{\dagger} \boldsymbol{n}_{l} .
\end{aligned}
$$

where the term $l^{\prime \prime} \in \mathcal{L}_{j}$ defined as the user of the other SBSs $j$ on sub-channel $k$, except for the SBSs in the coalition $S$.

Note that the remaining interference from other coalitions' SBSs, which reuse the same sub-channel, still affect the SBSs. In addition, the deployment of IA is accompanied by the cost of information exchange, complexity, and coordination, which highly limits the practical implementation. The achievable rate
$R_{n l}^{k}$ of receiver $l$ served by SBSs $n$ is given by (6) under the IA feasibility conditions:

$$
R_{n l}^{k}=\log \left(1+\alpha_{n l}^{k} \beta_{n l}^{t} P_{n l} \frac{\left(\boldsymbol{U}_{l}^{k}\right)^{\dagger} \boldsymbol{H}_{n l}^{k} \boldsymbol{V}_{n}^{k}\left(\boldsymbol{V}_{n}^{k}\right)^{\dagger}\left(\boldsymbol{H}_{n l}^{k}\right)^{\dagger} \boldsymbol{U}_{l}^{k}}{\left(\boldsymbol{U}_{l}^{k}\right)^{\dagger} \boldsymbol{B}_{l} \boldsymbol{U}_{l}^{k}}\right),
$$

where the term $\boldsymbol{B}_{l}$ is given by:

$$
\boldsymbol{B}_{l}=\sum_{j \in \mathcal{N} \backslash S} \alpha_{j l^{\prime \prime}}^{k} \beta_{j l^{\prime \prime}}^{t} P_{j l^{\prime \prime}} \boldsymbol{H}_{j l}^{k} \boldsymbol{V}_{j}^{k}\left(\boldsymbol{V}_{j}^{k}\right)^{\dagger}\left(\boldsymbol{H}_{j l}^{k}\right)^{\dagger}+\boldsymbol{n}_{l},
$$

where the term $P_{n l}$ is the transmission power of SBS $n$ to SUE $l$. The sum-rate of SBS $n$ denotes as (8):

$$
\begin{aligned}
& x_{n}=\sum_{t \in T} \sum_{k \in \mathcal{K}_{n}} \sum_{l \in \mathcal{L}_{n}} R_{n l}^{k} \\
& =\sum_{t \in T} \sum_{k \in \mathcal{K}_{n}} \sum_{l \in \mathcal{L}_{n}} \log \left(1+\alpha_{n l}^{k} \beta_{n l}^{t} P_{n l} \frac{\left(\boldsymbol{U}_{l}^{k}\right)^{\dagger} \boldsymbol{H}_{n l}^{k} \boldsymbol{V}_{n}^{k}\left(\boldsymbol{V}_{n}^{k}\right)^{\dagger}\left(\boldsymbol{H}_{n l}^{k}\right)^{\dagger} \boldsymbol{U}_{l}^{k}}{\left(\boldsymbol{U}_{l}^{k}\right)^{\dagger} \boldsymbol{B}_{l} \boldsymbol{U}_{l}^{k}}\right) .
\end{aligned}
$$

\section{Joint Interference Management Problem}

Based on the defined system model and the above analysis, we can observe that many users suffer from serious interference especially in the hotspots. In order to maximize the system performance in terms of the overall throughput, it is necessary to manage the interference by deploying the joint multi-domain interference management technologies. Moreover, in a resource management process, the related signaling overhead mainly includes the power consumption of pilot signals to estimate channel information, the overhead of user' feedback information, and the overhead of the interact information between the small base stations. To facilitate modeling, the power consumption of the pilot signal is used to estimate the cost of cooperation in this paper. Hence, by assuming $d=1, \forall t \in T, \forall k \in \mathcal{K}_{n} \subset \mathcal{K}$, the total throughput maximization problem (P1) is established as (9).

Where $\boldsymbol{\alpha}=\left[\alpha_{n l}^{k}\right] \in \mathbb{C}^{N \times L \times K}, \boldsymbol{\beta}=\left[\beta_{n l}^{k}\right] \in \mathbb{C}^{N \times L \times K}$ and $\boldsymbol{P}=\left[P_{n l}\right] \in \mathbb{C}^{N \times L}$ denotes the matrix of related elements. $\Phi$ is coalition sets of SBS, $P_{\max }$ and $\bar{P}_{S}$ are the maximum transmission power and the power cost of forming a coalition respectively. $P_{\lim }$ is the power consumption threshold of the coalition member transmitting the pilot tone to the furthest user in this coalition. Constraint (9b) represents the co-tier interference from other coalitions' SBS which reuses the same sub-channels. Constraint (9c) ensures each SUE can only use one sub-channel at a given time. Constraint (9d) guarantees each transmission pair is active in one time-slot. Constraint (9e) ensures the IA condition feasibility, and (9f) assures the cost of cooperation under a rational range. Constraints $(9 \mathrm{~g})$ and $(9 \mathrm{~h})$ give a reasonable transmission power range.

Unfortunately, the total throughput maximization problem (P1) over multiple variables is a non-convex optimization problem. Such a problem is intractable to solve directly and deployed with a centralized method. Thus, we solve the problem (P1) by decomposing it into four subproblems with distributed methods from multiple domain perspectives. By 


$$
\begin{gathered}
(\mathrm{P} 1) \max _{\boldsymbol{\alpha}, \boldsymbol{\beta}, \Phi, \boldsymbol{P}} \sum_{t \in T} \sum_{n \in \mathcal{N}} \sum_{k \in \mathcal{K}_{n}} \sum_{l \in \mathcal{L}_{n}} R_{n l}^{k}=\sum_{t \in T} \sum_{n \in \mathcal{N}} \sum_{k \in \mathcal{K}_{n}} \sum_{l \in \mathcal{L}_{n}} \log \left(1+\alpha_{n l}^{k} \beta_{n l}^{t} P_{n l} \frac{\left(\boldsymbol{U}_{l}^{k}\right)^{\dagger} \boldsymbol{H}_{n l}^{k} \boldsymbol{V}_{n}^{k}\left(\boldsymbol{V}_{n}^{k}\right)^{\dagger}\left(\boldsymbol{H}_{n l}^{k}\right)^{\dagger} \boldsymbol{U}_{l}^{k}}{\left(\boldsymbol{U}_{l}^{k}\right)^{\dagger} \boldsymbol{B}_{l} \boldsymbol{U}_{l}^{k}}\right) \\
\boldsymbol{B}_{l}=\sum_{j \in \mathcal{N} \backslash S} \alpha_{j l^{\prime \prime}}^{k} \beta_{j l^{\prime \prime}}^{t} P_{j l^{\prime}} \boldsymbol{H}_{j l}^{k} \boldsymbol{V}_{j}^{k}\left(\boldsymbol{V}_{j}^{k}\right)^{\dagger}\left(\boldsymbol{H}_{j l}^{k}\right)^{\dagger}+\boldsymbol{n}_{l}, \\
\text { s.t. : } \sum_{k \in \mathcal{K}_{n}} \alpha_{n l}^{k}=1, \forall n \in \mathcal{N}, \mathcal{K}_{n} \in \mathcal{K}, \\
\sum_{t \in T} \beta_{n l}^{t}=1, \forall n \in \mathcal{N}, \\
|S| \leq N_{t}+N_{r}-1, \forall S \subset \Phi \subset \mathcal{N}, \\
\bar{P}_{S} \leq P_{\lim }, \forall S \subset \Phi, k \in \mathcal{K}, \\
P_{n l} \geq 0, \forall n \in \mathcal{N}, l \in \mathcal{L}_{n}, \\
\sum_{l \in L_{n}} P_{n l} \leq P_{\max }, \forall n \in \mathcal{N},
\end{gathered}
$$

solving the subproblems one by one, we finally obtain a suboptimal solution of (P1) in the end.

\section{Joint InTERFERENCE MANAGEMENT IN Ultra-DENSE SMALl NeTWORKS}

We propose a distribution JIM algorithm to solve the problem (P1) in this section, with its flowchart shown in Fig. 2. In order to maximize spectrum utilization and considering complexity, we implement OFDMA scheduling to reduce the neighboring co-tier interference in one slot at first, where the local subcarrier allocation matrix parameter $\boldsymbol{\alpha}$ is solved in the frequency domain. Next, because the cooperation cost among SBSs from the intra-coalition is less than the SBSs from the inter-coalition, the appropriate overlapping coalitions of SBSs is formed to deploy IA over the interference links to reduce the intra-coalition interference. Thus, the parameter $\Phi$ is solved in the space domain, where the practical limits such as cooperative cost and IA feasibility conditions are taken into consideration. Then, the residual inter-coalition interference is suppressed by TDMA scheduling further, i.e., the parameter $\boldsymbol{\beta}$ TDMA time-slots allocation matrix is solved in the time domain. Finally, the transmission power is optimized by the distributed parallel iterative water-filling algorithm in each SBS to eliminate the residual interference, i.e., the parameter $\boldsymbol{P}$ is solved in the power domain. In the following pages, we separately introduce the four subproblems of JIM.

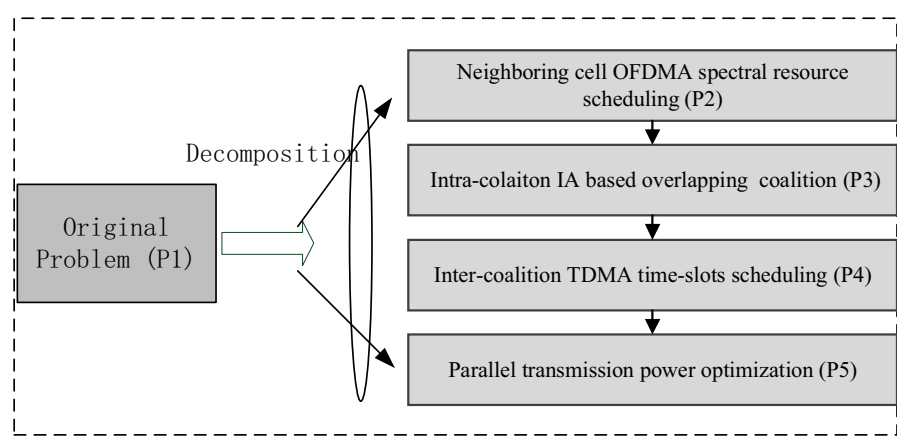

Fig. 2. Primal problem is decomposed into four subproblems.

\section{A. OFDMA Spectral Resource Scheduling (Phase I)}

Because the IA must be performed in a certain transmission link, we first find the proper sub-channel allocation that should be aligned for each receiver within the IA feasibility constraint. In other words, we solve $\alpha$ of the primal problem (P1) from the spectral domain perspective. i.e., optimizing the subproblem (P2):

$$
\begin{aligned}
& \text { (P2) } \max _{\alpha} \sum_{n \in \mathcal{N}} \sum_{k \in \mathcal{K}_{n}} \sum_{l \in \mathcal{L}_{n}} R_{n l}^{k} \\
& =\sum_{n \in \mathcal{N}} \sum_{k \in \mathcal{K}_{n}} \sum_{l \in \mathcal{L}_{n}} \log \left(1+\alpha_{n l}^{k} P_{n l} \frac{\left(U_{l}^{k}\right)^{\dagger} \boldsymbol{H}_{n l}^{k} \boldsymbol{V}_{n}^{k}\left(\boldsymbol{V}_{n}^{k}\right)^{\dagger}\left(\boldsymbol{H}_{n l}^{k}\right)^{\dagger} \boldsymbol{U}_{l}^{k}}{\left(\boldsymbol{U}_{l}^{k}\right)^{\dagger} \boldsymbol{B}_{l} \boldsymbol{U}_{l}^{k}}\right) \\
& \boldsymbol{B}_{l}=\sum_{j \in \mathcal{N} \backslash S, j \neq n} \alpha_{j l^{\prime \prime}}^{k} P_{j l^{\prime \prime}} \boldsymbol{H}_{j l}^{k} \boldsymbol{V}_{j}^{k}\left(\boldsymbol{V}_{j}^{k}\right)^{\dagger}\left(\boldsymbol{H}_{j l}^{k}\right)^{\dagger}+\boldsymbol{n}_{l}, \quad(10 \mathrm{a}) \\
& \quad \text { s.t. : } \sum_{k \in \mathcal{K}_{n}} \alpha_{n l}^{k}=1, \forall n \in \mathcal{N}, \mathcal{K}_{n} \in \mathcal{K}, \quad(10 \mathrm{c})
\end{aligned}
$$

where other related variables will remain the same in this subsection. Thus, we present a simple and sub-optimal distributed approach to address the problem by the OFDMA scheduling based on the signal to interference plus noise ratio (SINR). The details of OFDMA scheduling can be found in the JIM algorithm.

The proper OFDMA sub-channel set can be allocated to avoid strong interference from neighboring SBSs. However, because of dense deployment of SBSs and limited spectrum resource, the OFDMA resource allocation cannot solve the severe co-tier interference. Thus, we further solve the interference problem by the IA in the space domain.

\section{B. Small Cell Cooperation as OCFG to Perform IA (Phase II)}

IA is one of the powerful cooperative transmission techniques that effectively mitigates interference and improves the overall capacity [34]-[36]. To further reduce the interference by IA from the space domain, we model the SBSs' cooperation behavior as an OCFG to form coalitions that satisfies the practical limits. In other words, we further solve the interference 
subproblem (P3):

$$
\begin{gathered}
(\mathrm{P} 3) \max _{\Phi} \sum_{n \in \mathcal{N}} \sum_{k \in \mathcal{K}_{n}} \sum_{l \in \mathcal{L}_{n}} R_{n l}^{k} \\
\text { s.t. : }|S| \leq N_{t}+N_{r}-1, \forall S \subset \Phi \subset \mathcal{N} \\
\bar{P}_{S} \leq P_{\lim }, \forall S \subset \Phi, k \in \mathcal{K} .
\end{gathered}
$$

To perform IA with the proper coalitions which satisfy the IA feasibility conditions, some basic definitions of OCFG are introduced to provide a foundation framework. In [26], OCFG as a novel cooperative framework has been presented. Its main characteristic was that the players can participate in multiple coalitions. The goal of (P3) is to maximize the total throughput while taking into account the cooperation costs. The related definitions are given as follows:

Definition 1: An OCFG with a transferable utility (TU) is defined by a pair $G=(\mathcal{N}, v, \Phi)$. Here $\mathcal{N}$ represents a set of players, $v$ is a value function that assigns a real value to each coalition, and coalitional structure $\Phi$, defining a set of coalitions. A set of coalitions $\Phi=\left(S_{1}, \ldots, S_{r}, S_{n} \ldots, S_{R}\right), S_{r} \cap$ $S_{n} \neq \emptyset, \exists r \neq n, S_{r}, S_{n} \subset \Phi$, where each coalition consists of SBSs which reuse the same sub-channel and $S_{r}$ is the $r$-th coalition.

Note that $v(\emptyset)=0$. The TU property means that the total utility can be divided by the coalition members in any manner. Furthermore, each SBS in a coalition reuses the same frequency at least once.

Definition 2: The term $\lambda_{n}^{k}$ represents the minimum invisible resource unit of SBSs, i.e., a single sub-channel $k$ from $\mathcal{K}_{n}$. Any resource unit can switch to a new coalition with a preferred coalitional by SBS. Similarly, the term $\lambda_{n}^{k} \in S_{r}$ is an SBS unit which means the unit $\lambda_{n}^{k}$ belong to the coalition $S_{r}$, where the number of coalition reuse the same sub-channel $k$. To facilitate the understanding, $S_{r}^{k}$ is defined as the coalition $S_{r}$ in the sub-channel $k$.

The cooperation of SBSs brings remarkable benefits, but also accompanies by an inherent cost such as the information exchange. Because the operation involves all SUEs in the coalition, it is acceptable to assume that the amount of transmit power can be captured as the cost of forming coalitions. Hence, for a given partial coalition $S_{r}^{k}$, each SBS $n \in S_{r}^{k}$ requires to broadcast the related information to farthest SBS $i^{*} \in S_{r}^{k}$ in the same coalition. The power cost of forming a coalition is denoted as follow:

$$
\begin{gathered}
\bar{P}_{S_{r}^{k}}=\sum_{n \subset S_{r}^{k}} P_{n, i^{*}}^{k} \leq P_{\lim } \\
P_{n, i^{*}}^{k}=\frac{v_{\min } \times \delta_{n}^{2}}{\left|H_{n, i^{*}}^{k}\right|^{2}}
\end{gathered}
$$

where the term $v_{\text {min }}$ denotes the minimum SINR required at the potential coalition partner $i^{*}$. The term $\left|H_{n, i^{*}}^{k}\right|^{2}$ denotes the channel gain between SBS $n$ and SBS $i^{*}$ over the common control channel. The term $\delta_{n}^{2}$ denotes the noise power. SBSs act as the player that can join more than one coalitions in
OCFG $G=(\mathcal{N}, v)$ to obtain a better performance. The value of coalition $S_{r}$ is denoted as follow:

$$
v\left(S_{r}, \Phi\right)=\left\{\begin{array}{l}
\sum_{n \in S_{r}} R_{n l}^{k}, \quad \text { if } \quad \bar{P}_{S_{r}} \leq P_{\lim } \\
0, \quad \text { otherwise. }
\end{array}\right.
$$

Consequently, the value of $\Phi$ is expressed as

$$
v(\Phi)=\sum_{S_{r} \subset \Phi} v\left(S_{r}, \Phi\right) .
$$

Note that $v(\Phi)$ denotes the system's payoff. When an SBS $n \in \mathcal{N}$ negotiates with the potential coalition partner to decide whether to cooperate over the sub-channel $k$, there exist two conditions: (i) SBS $n$ deviates from the original coalition and participates in the potential coalition; (ii) SBS $n$ stays in the original coalition.

Definition 3: Consider a coalitional structure define as $\Phi=$ $\left\{S_{1}{ }^{k}, \ldots, S_{r}{ }^{k}, \ldots, S_{n}{ }^{k}, \ldots S_{R}{ }^{k}\right\}$, where an SBS unit $\lambda_{n}^{k}$ satisfies $\lambda_{n}^{k} \in S_{r}^{k}$. A new coalitional structure can be defined as $\Phi^{\Delta}=$ $\left\{\Phi \backslash\left\{S_{r}^{k}, S_{n}^{k}\right\}\right\} \cup\left\{S_{r}^{k} \backslash\left\{\lambda_{n}^{k}\right\}\right\} \cup\left\{S_{n}^{k} \cup\left\{\lambda_{n}^{k}\right\}\right\}$. The SBS $n$ unit $\lambda_{n}^{k}$ that switches from $S_{r}^{k}$ to $S_{n}^{k}$ must meet the transfer rule $\triangleright$. The transfer rule is denoted as follow:

$$
\Phi \triangleright \Phi^{\Delta} \Leftrightarrow\left\{\begin{array}{l}
x_{n}\left(\Phi^{\Delta}\right)>x_{n}(\Phi) \\
v\left(S_{n}, \Phi^{\Delta}\right) \geq v\left(S_{r}, \Phi\right) \\
v\left(\Phi^{\Delta}\right)>v(\Phi) \\
(4),(12) \text { are satisfied. }
\end{array}\right.
$$

By the transfer rule, when an SBS unit performs the switching operation, four conditions are needed: (i) the individual utility of SBS $n$ is increased, (ii) the value of newly formed coalition $S_{n}$ does not decrease, (iii) the total utility of the new $\Phi^{\Delta}$ is increased. (iv) two constraints (4) and (12) should be satisfied.

Definition 4: An outcome $(\Phi, v)$ is stable if no unit of player $n \in \mathcal{N}$ has a profitable switching operation.

There is little impact on the overall system performance of coalitional structure with a trivial change in USNs. Hence, the convergence of the coalitional structure can be estimated by a minimum judgment threshold to reduce the iteration, such as (17).

$$
\left|v(\Phi)-v\left(\Phi^{*}\right)\right| /|v(\Phi)| \leq v_{t h} .
$$

To facilitate the implementation of the proposed JIM algorithm and achieve a stable overlapping coalitional structure, we assume that the minimum threshold $v_{t h}$ is sufficiently small, and the SBS can collect all received signal strength indication (RSSI) and channel state information (CSI) of neighboring SBSs. Furthermore, the term $h\left(\lambda_{n}^{k}\right)$ denotes the history set of the SBS unit $\lambda_{n}^{k}$, where the past of coalition is recorded.

Proposition 1: Given the transfer rule, the convergence of the final coalitional structure $C S^{*}$ is guaranteed in the OCFG.

Proof: There exists a resource unit $\lambda_{n}^{k}$ if the final coalitional structure $\Phi^{*}$ does not converge, which can be transferred from the current coalitional structure $\Phi^{*}$ into a new coalitional structure $\Phi^{\Delta}$ with a profitable switching. Hence, the difference of the total payoff between two coalitional structures cannot satisfy the minimum judgment threshold. This contradicts the 
fact that $\Phi^{*}$ is no convergent. Hence, the final coalitional structure is convergent.

\section{Inter-coalition Time Slots Scheduling (Phase III)}

As mentioned previously, each SBS can form the overlapping coalitions. There still exists interference among the coalitions which reuse the same sub-channel. Thus, we further address the inter-coalition interference by using the TDMA scheduling.

Based on the formed overlapping coalition structure, we choose a representative SBS among each coalition which suffers the most from the coalition member residual interference. The subproblem can be reduced to an optimization problem in multi-user TDMA scheduling, i.e., optimizing the sub-problem (P4):

$$
\begin{gathered}
\max _{\beta} \sum_{t \in \mathcal{T}} \sum_{S_{r} \subset \Phi} \sum_{n \in S_{r}} \beta_{n l}^{t} x_{n} \\
\sum_{t \in \mathcal{T}} \beta_{n}^{t}=1, \forall n \in \mathcal{N}, S^{r} \subset \Phi^{*} .
\end{gathered}
$$

\section{Transmission Power Optimization (Phase IV)}

Finally, each SBS updates its transmission power using the water-filling algorithm in [29], which is dependent on the interference information measured in previous iterations, i.e., to solve the subproblem (P5):

$$
\begin{gathered}
\max _{\boldsymbol{P}} \sum_{n \in \mathcal{N}} \sum_{l \in \mathcal{L}_{n}} R_{n l}^{k} \\
=\sum_{n \in \mathcal{N}} \sum_{l \in \mathcal{L}_{n}} \log \left(1+P_{n l} \frac{\left(\boldsymbol{U}_{l}^{k}\right)^{\dagger} \boldsymbol{H}_{n l}^{k} \boldsymbol{V}_{n}^{k}\left(\boldsymbol{V}_{n}^{k}\right)^{\dagger}\left(\boldsymbol{H}_{n l}^{k}\right)^{\dagger} \boldsymbol{U}_{l}^{k}}{\left(\boldsymbol{U}_{l}^{k}\right)^{\dagger} \boldsymbol{B}_{l} \boldsymbol{U}_{l}^{k}}\right) \\
\text { s.t. }: P_{n l} \geq 0, \forall n \in \mathcal{N}, l \in \mathcal{L}_{n}, \\
\sum_{l \in L_{n}} P_{n l} \leq P_{n \max }, \forall n \in \mathcal{N} .
\end{gathered}
$$

\section{E. The Proposed JIM Algorithm}

To summarize, the proposed JIM algorithm in this paper is presented by Algorithm 1. It is worth noting that the signals involved in the algorithm includes: pilots, CSI feedbacks, signaling among small cell base stations and so on. For example, the SUE should send the interference information from the neighboring SBSs to its own SBS; In the coalition formation process, the SBS needs to send the cooperation request to the potential partner, and the requested SBS needs to feed back the result of the request.

\section{F. Complexity}

In this subsection, we analyze the complexity of the proposed JIM algorithm. As mentioned above, the whole JIM algorithm includes four parts, i.e., OFDMA scheduling, IA, TDMA scheduling, and power control. Let $L$ represent the maximum number of SUEs for all small cell. The complexity of the JIM algorithm for each SBS is given as follow:

$$
\mathrm{O}(\underbrace{K L}_{(a)}+\underbrace{N L}_{(b)}+\underbrace{L T}_{(c)}+\underbrace{L}_{(d)})
$$

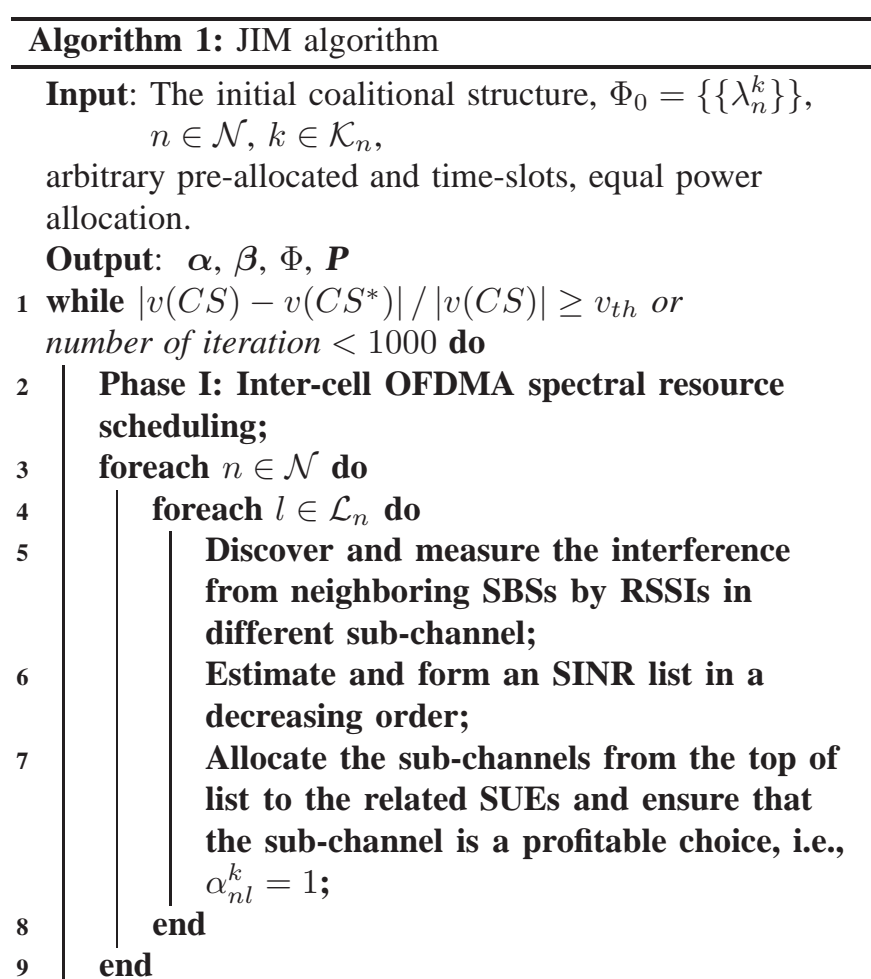

Phase II: Intra-coalition IA based overlapping coalitions;

foreach $n \in \mathcal{N}$ do 
where part (a) is the worse case complexity for SBS. Part (b) is the worse case complexity of the coalition formation and the related IA matrices design for each SBS. Part (c) is the worse case of the TDMA scheduling for each SBS. Part (d) is the complexity of the power control. Moreover, it is worth pointing out that the sensitivity of the algorithm's phase to the channel estimation error is different. Specifically, phase III is poorer than other phases, due to the zero-forcing suppression matrix of interference alignment techniques relying on the channel state information of the receivers' feedback.

Moreover, The upper bound of the total throughput can be obtained by using the exhaustive search method, except for the power control. The worse case complexity of the exhaustive search method is $\mathrm{O}\left(K T L^{2}(N-1)^{(N L)}\right)$. Since the exhaustive search method has exponential computational complexity, and it is not practical for a large-scale network. Furthermore, the complexity of the work [7]'s method is $\mathrm{O}(K N L)$ in the worse case, the main reason is the user maybe traverses all base station and resource block. Thus, compared with the exhaustive search method and the work [7]'s method, our proposed algorithm has a lower complexity.

\section{Practical Deployment and ApPlication}

In this section, with some reasonable assumptions, we illustrate the practical implementation of the proposed JIM scheme. The related hypotheses are as follows:

1 The sub-channels are enough for the users and one user occupied one sub-channel at one time in each small cell.

2 Each SBS collects the neighboring channels/users information by its own SUE and exchanges information in order to execute dynamically in real time.

3 The wired/wireless backhaul among the SBSs is enough for the information exchange.

Given the above assumptions, a possible way to implement the proposed JIM algorithm is as follow:

The initial coalitional structure consists of non-cooperation SBSs. Thus, there exists a severe co-tier interference problem in the UDNs. First, each SBS $n$ delivers a pilot tone to acquire the CSI estimation of the neighboring SBSs by the active SUE. Each SUE receives the pilot tone and estimates its local CSI of the effective channel as well as the interference channels. The obtained CSI of each SUE would serve as a fed back to the related SBS. Each SBS discovers the neighboring SBSs and collects the RSSIs by its own active SUEs. Based on the measured interference information, different descending SINR list is formed in each sub-channel by the SBS. Then, each SBS distributes the proper sub-channels from the top of the list for its own SUEs. Second, the new interference state is estimated at each SBS, while the interference list in a decreasing order is updated. Based on the interference list, each SBS selects the potential coalition partners and judges whether to cooperate through the history set and transfer rule. In each coalition, the IA operation has been deployed under the IA feasibility conditions. Third, each coalition selects a representative SBS to measure the other coalitions' interference. Then. the representative SBS forms an ascending interference list of neighboring representative SBSs with different time-slots.
Each representative SBS selects a better time-slot from the top of the list for the coalition. Finally, the power optimization is implemented by the water-filling algorithm. This process is repeated until the set judgment threshold or an iteration limit is satisfied, i.e., convergence. Through the above process, the co-tier interference is reduced gradually from multi-domain.

\section{Simulation Results and Analysis}

Simulation is conducted using Matlab 2014b to estimate the proposed algorithm in terms of total throughput, coalition size, and so on. Let us consider a network system, where multiple SBSs are randomly and uniformly located in a single hexagonal macrocell. The hexagonal radius of MBS and SBS are $500 \mathrm{~m}$ and $20 \mathrm{~m}$, respectively. In each small cell, four SUEs are uniformly distributed throughout the cell. The total number of OFDMA sub-channels is 12 in each SBS. The total number of time slots is 4 in each transmission in TDMA mode. The maximum transmit power of SBS and SUE are $23 \mathrm{dBm}$ and 20 $\mathrm{dBm}$, respectively. The noise variance is set to $-174 \mathrm{dBm} / \mathrm{Hz}$. Simulation methodologies and parameters typically used to evaluate LTE-A systems have been adopted from [30]. The final results are averaged over many simulations to leverage the channel variations.

TABLE I

SIMULATION PARAMETERS

\begin{tabular}{|c|c|}
\hline \hline Parameter & Value \\
\hline Coverage radius of MBS & $500 \mathrm{~m}$ \\
Coverage radius of SBS & $20 \mathrm{~m}$ \\
Carrier frequency & $2.0 \mathrm{GHz}$ \\
Total Bandwith & $20 \mathrm{MHZ}$ \\
Subcarrier Bandwidth & $180 \mathrm{KHz}$ \\
Maximum BS Transmit power & $20 \mathrm{dBm}$ \\
Maximum UE Transmit power & $23 \mathrm{dBm}$ \\
Number of User & 4 \\
Antenna Configurations & 2 \\
Path Loss (dB) d in KM & $140+37.6 \log 10(\mathrm{~d})$ \\
UE Antenna Gain & $0 \mathrm{dBi}$ \\
BS Antenna Gain + Cable Loss & $5 \mathrm{dBi}$ \\
Thermal Noise density & $-174 \mathrm{dBm} / \mathrm{Hz}$ \\
\hline
\end{tabular}
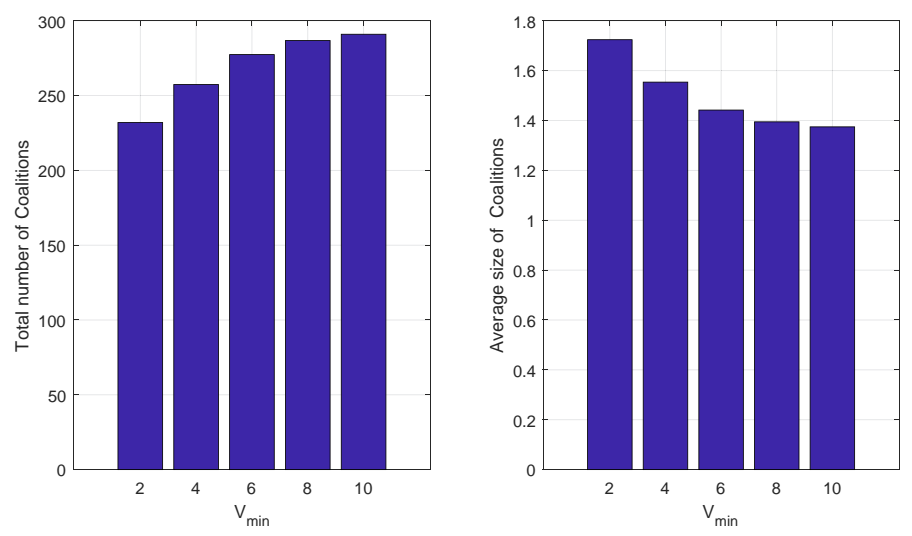

Fig. 3. Total number of coalitions and the average size of coalitions with different minimum SNRs $v_{\min }$.

In Fig. 3, we assume the minimum SINR $v_{\min }$ varying with the influence of the total number of coalitions and the average size of coalitions, where the number of SBSs is 100 , 
the maximum tolerable power cost $P_{\text {lim }}$ is $23 \mathrm{dBm}$, and the judgment threshold $v_{t h}$ is 0.005 . There initially consists of the non-cooperative SBSs. The initial coalitional structure is the minimum invisible resource unit. With the increasing $v_{\text {min }}$, the number of coalitions increases, the size of coalition decreases. The reason is that the increased minimum SINR always leads to a large cost of cooperation, i.e., the cost of information exchange increases along with the increasing minimum SINR. Thus, forming coalitions among the SBSs becomes satisfactory. In short, Fig. 3 shows that the minimum SINR affects the coalition formation with the cost of information exchange. Moreover, the minimum SINR decides the farthest distance of the neighboring SBSs.
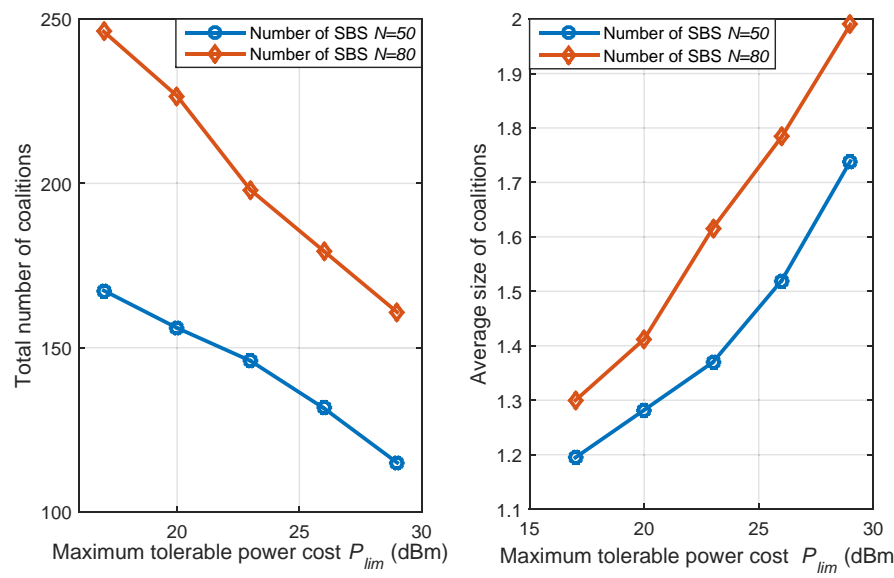

Fig. 4. Total number of coalitions and the average size of coalitions with different maximum tolerable power cost $P_{\text {lim }}$.

Meanwhile, Fig. 4 shows the total number of coalitions and the average size of coalitions with the target maximum tolerable power cost $P_{\text {lim }}$, where the number of SBS is set to be 50 and 80 , the $v_{\min }$ is $3 \mathrm{~dB}$, and the judgment threshold $v_{t h}$ is 0.005 . The tendency of improvement in Fig. 3 is opposite to Fig. 4. As the maximum tolerable power cost increases, the total number of coalitions decreases, and the average size of coalition increases. Fig. 4 indicates that the SBSs have more incentives to form coalitions with the increase of maximum tolerable power cost.

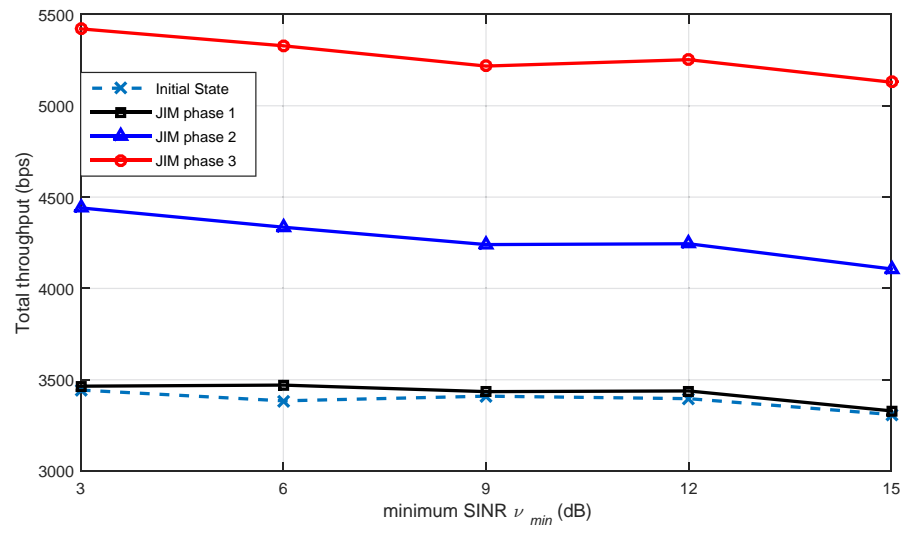

Fig. 5. Total throughput with different minimum SINR $v_{m i n}$

Fig. 5 shows the total throughput of different JIM phases with the varying minimum SINR $v_{\min }$. Here, it is known that the $v_{\min }$ affects the farthest distance of the neighboring SBSs and the coalition formation. Thus, the four phases of the JIM algorithm are illustrated in Fig. 5. The initial state is the random network state, i.e., all SBSs pre-allocated OFDMA sub-channels arbitrary and reuse the same time-slots for its user, and the initial power allocation is equal. The initial coalitional structure is denoted as the minimum invisible resource unit set $\left\{\lambda_{n}^{k}\right\}, n \in \mathcal{N}, k \in \mathcal{K}_{n}$. Phase I is the OFDMA scheduling of the JIM algorithm, which decreases with the increasing minimum SINR. The main reason for that is $v_{\text {min }}$ decides the area of the neighboring SBSs. Phase II includes OFDMA scheduling and coalition IA, and the total throughput changes with the cost of information exchanges. Similarly, the performance in Phase III will be further improved with the area of neighboring SBSs.

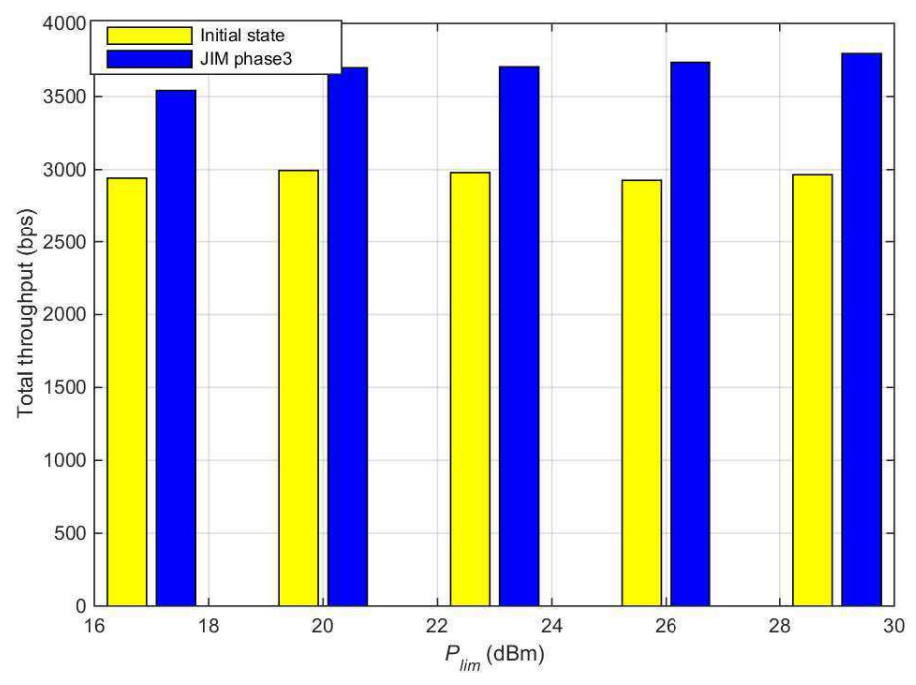

Fig. 6. The total throughput with different maximum tolerable power cost $p_{\text {lim }}$.

Fig. 6 shows the total throughput with the maximum tolerable power cost. The coalition formation is affected by the maximum tolerable power cost. Thus, we estimate the total throughput of Phase III of the proposed JIM algorithm, compared to the initial state. We conclude that the higher maximum tolerable power cost allows more potential partners to cooperatively form a coalition. Meanwhile, each coalition satisfies the IA feasibility conditions of (4). Similar to Fig. 4 , the total throughput of Phase III of the presented JIM algorithm increases with the increasing maximum tolerable power cost. 


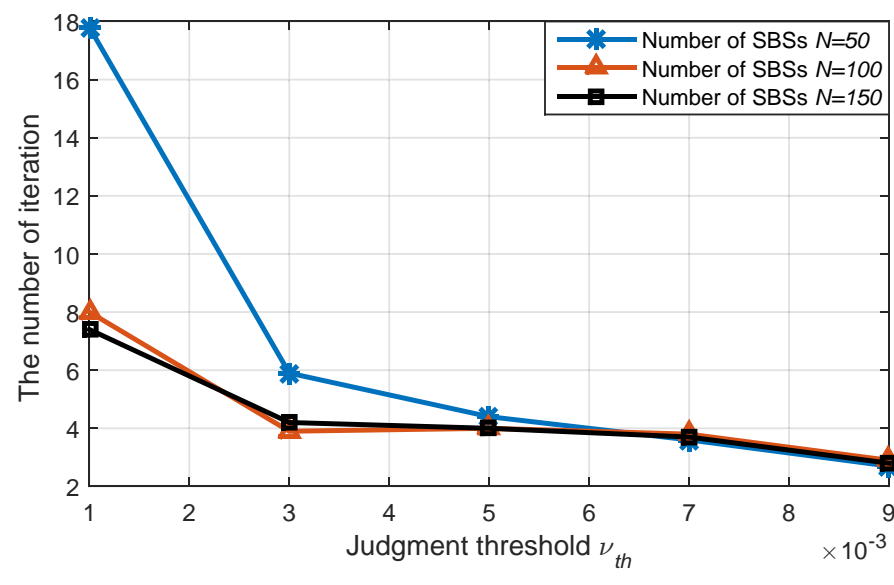

Fig. 7. The number of iterations with different threshold $v_{t h}$.

Fig. 7 depicts the number of iteration as a function of the judgment threshold with different number of SBS $N=$ $\{50,100,150\}$. Here, we set the $v_{\text {min }}=3 \mathrm{~dB}$, the $p_{\text {lim }}=17$ $\mathrm{dBm}$. We conclude that different thresholds affect the number of iterations. In particular, the number of iterations decreases rapidly because of the improvement of total throughput which is more remarkable in larger networks when the judgment threshold and the number of SBSs are large.
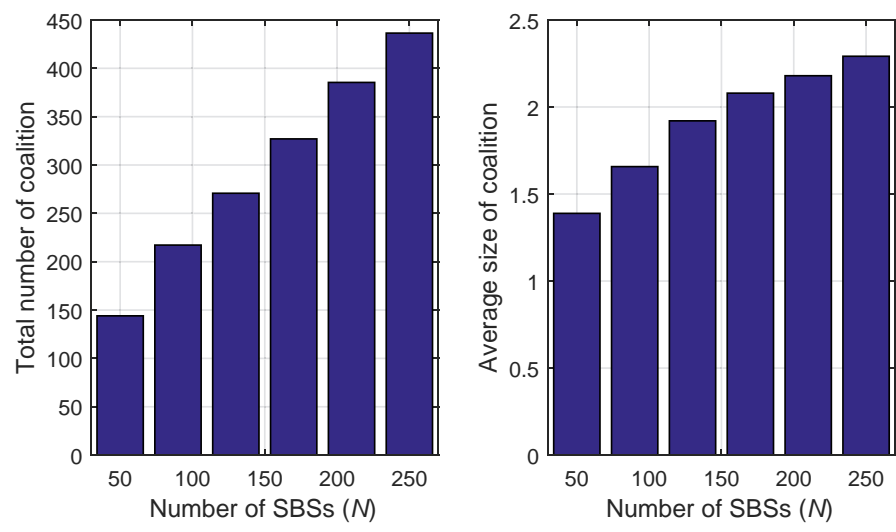

Fig. 8. Total number of coalitions and the average size of coalitions with increasing number of SBSs.

A total number of coalitions and the average size of coalitions with the increasing number of SBSs is shown in Fig. 8. It can be seen that the spatial separation of SBSs brings about a significant cost of cooperation, resulting in the coalition rarely formed when the density of networks is small. Meanwhile, more SBSs have conflict transmission over the same sub-channel with an interference level and an increase of SBSs' density. The SBS will have an incentive to deviate towards forming cooperations, and thus more SBSs can utilize the coalition to coordinate their transmissions. Moreover, the maximum size of the coalition is constrained by the cost of cooperation as well as the inherent IA condition (i.e., the IA feasibility conditions such as: $N_{t}+N_{r} \geq|S|+1$, when $d=1$ ). The bigger the coalition size, the higher number of antennas required.

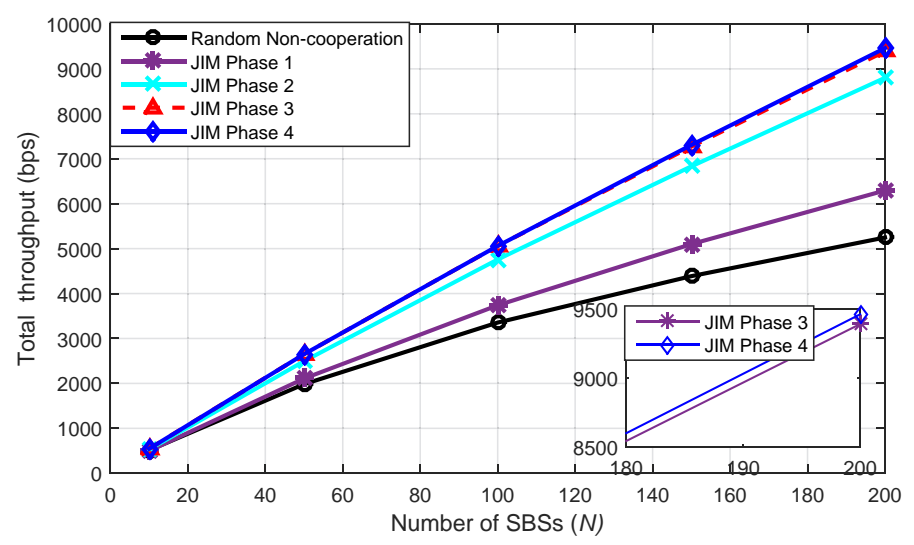

Fig. 9. Total throughput as number of SBSs increases.

Fig. 9 shows the total throughput as a function of the number of SBSs. Different phases of the proposed JIM algorithm are compared with a random non-cooperative scheme. The random non-cooperative scheme randomly allocates the subchannels and time-slots to users. Obviously, the improvement of power optimization is almost invisible. The main reason is that the power is similar to equal allocation when applying the water-filling algorithm in the high SINR region. As the number of SBSs grows, the total throughput increases and the performance of the proposed JIM scheme becomes much better than the non-cooperative scheme. Moreover, the gains between the random non-cooperation schemes and JIM algorithm are enlarged. When the number of SBS reaches 200, the improvement in terms of total throughput is approximately up to $80 \%$ by the JIM scheme, compared to the random noncooperation scheme. Furthermore, the increasing rate of the total throughput decreases. The main reason is that the residual interference among the coalitions increases with the limited spectrum resources. Meanwhile, the improvement of power optimization begins with the decreasing SINR.

\section{CONCLUSION}

In this paper, we have jointly investigated the interference management techniques of different domains in USNs. A JIM algorithm has been proposed to deal with the co-tier interference of SBSs, which includes OFDMA scheduling, IA, TDMA scheduling, and power optimization. We first implemented the OFDMA scheduling to allocate the proper subchannels and establish the stable transmission links to reduce the interference from neighboring SBSs. Then, based on the OCFG, the SBSs constituted a stable overlapping coalition structure, where the intra-coalition interference is aligned by IA. Followed by reducing the co-tier interference among coalitions is reduced with TDMA scheduling. Finally, the residual interference is mitigated by the power optimization further. The optimizing total throughput is acquired by repeating the above steps. By using this distributed algorithm, each SBS can interact and make decisions independently to form the coalition and reduce the interference from different domains, thus realizing the optimal tradeoff between costs and benefits. The proposed JIM algorithm outperformed other approaches as demonstrated via simulation results. 


\section{REFERENCES}

[1] M. Ding and D. Lopez-Perez, "On the performance of practical ultradense networks: The major and minor factors," 2017 15th International Symposium on Modeling and Optimization in Mobile, Ad Hoc, and Wireless Networks (WiOpt), Paris, France, 2017, pp. 1-8.

[2] D. Lopez-Perez, M. Ding, H. Claussen, and A. H. Jafari, "Towards 1 Gbps/UE in Cellular Systems: Understanding Ultra-Dense Small Cell Deployments," IEEE Communications Surveys \& Tutorials, vol. 17, no. 4, pp. 2078-2101, Fourthquarter 2015.

[3] C. Yang, J. Li, and M. Guizani, "Cooperation for spectral and energy efficiency in ultra-dense small cell networks," IEEE Wireless Соттиnications, vol. 23, no. 1, pp. 64-71, Feb. 2016.

[4] M. Kamel, W. Hamouda, and A. Youssef, "Ultra-dense networks: a survey," IEEE Communications Surveys \& Tutorials, vol. 18, no.4, pp. 2522-2545, Fourthquarter 2016.

[5] T. K. Vu, M. Bennis, S. Samarakoon, M. Debbah, and M. Latva-aho, "Joint Load Balancing and Interference Mitigation in 5G Heterogeneous Networks", IEEE Transactions on Wireless Communications, vol. 16, no. 9, pp. 6032-6046, Sept. 2017.

[6] TI. Shgluof, M. Ismail, and R. Nordin, "Semi-Clustering of VictimCells Approach for Interference Management in Ultra-Dense Femtocell Networks", IEEE Access, vol. 5, pp. 9032-9043, 2017.

[7] D. Qu, Y. Zhou, L. Tian, and J. Shi, "User-Centric QoS-Aware Interference Coordination for Ultra Dense Cellular Networks", 2016 IEEE Global Communications Conference (GLOBECOM), Washington, DC, 2016, pp. 1-6.

[8] G. J. Foschini, Z. Miljanic, "A simple distributed autonomous power control algorithm and its convergence", IEEE Transactions on Vehicular Technology, vol. 42, no. 4, pp. 641-646, Nov. 1993.

[9] V. Chandrasekhar, J. Andrews, Z. Shen, T. Muharemovic, and A. Gatherer, "Power control in two-tier femtocell networks", IEEE Transactions on Wireless Communications, vol. 8, no. 8, pp. 4316-4328, Aug. 2009.

[10] J. Y. Lee, S. J. Bae, Y. M. Kwon, and M. Y. Chung, "Interference analysis for femtocell deployment in OFDMA systems based on fractional frequency Reuse," IEEE Communications Letters, vol. 15, no. 4, pp. 425-427, Apr. 2011.

[11] N. Saquib, E. Hossain, and D. I. Kim,"Fractional frequency reuse for interference management in LTE-advanced hetnet", IEEE Wireless Communications, vol. 20, no. 2, pp. 113-122, Apr. 2013.

[12] J. Torregoza, R. Enkhbat, and W. J. Hwang, "Joint power control, base station assignment, and channel Assignment in Cognitive Femtocell Network", EURASIP Journal on Wireless Communications and Networking, vol. 2010, no.6, pp. 1-14, May. 2010.

[13] L. Giupponi and C. Ibars, "Distributed interference control in OFDMAbased femtocells," 21st Annual IEEE International Symposium on Personal, Indoor and Mobile Radio Communications, Instanbul, pp. 12011206, Sept. 2010.

[14] D. Lopez-Perez, A. Valcarce, G. de la Roche, E. Liuand, and J.Zhang, "Access methods to WiMAX femtocells: a downlink system-level case study", 2008 11th IEEE Singapore International Conference on Communication Systems, Guangzhou, 2008, pp. 1657-1662.

[15] A. K. Karmokar, S. Senthuran, and A. Anpalagan, "Physical layeroptimal and cross-layer channel access policies for hybrid overlayunderlay cognitive radio networks", IET Communications, vol. 8, no. 15 , pp. 2666-2675, Oct. 2014

[16] S. Randan and R. Madan, "Belief propagation methods for intercell interference coordination in femtocell networks", IEEE Journal on Selected Areas in Communications, vol. 30, no. 3, pp. 631-640, Apr. 2012.

[17] D. Lopez-Perez, I. Guvenc, G. de la Roche, M. Kountouris, T. Q. S. Quek, and J. Zhang, "Enhanced intercell interference coordination challenges in heterogeneous networks", IEEE Wireless Communications, vol. 18 , no. 3 , pp. $3425-3441$, Aug. 2008.

[18] V. R. Cadambe and S. A. Jafar, "Interference alignment and degrees of freedom of the k-user interference channel", IEEE Transactions on Information Theory, vol. 54, no. 8, pp. 3309-3322, Jun. 2011.

[19] Pantisano F, Bennis M, Saad W, et al. "Interference alignment for cooperative femtocell networks: a game-theoretic approach," IEEE Transactions on Mobile Computing, vol. 12, no. 11, pp. 2233-2246, Nov. 2013.

[20] J. G. Andrews, X. Zhang, G. D. Durgin, and A. K. Gupta, "Are we approaching the fundamental limits of wireless network densification?," CoRR, vol. abs/1512.00413, 2015. [Online]. Available: http://arxiv.org/abs/1512.00413
[21] F. Pantisano, M. Bennis, W. Saad, R. Verdone, and M. Latva-aho, "Coalition formation games for femtocell interference management: a recursive core approach", 2011 IEEE Wireless Communications and Networking Conference, Cancun, Quintana Roo, 2011, pp. 1161-1166.

[22] C. Yang, J. Li, Q. Ni, A. Anpalagan, and M. Guizani, "Interferenceaware energy efficiency maximization in $5 \mathrm{G}$ ultra-dense networks," IEEE Transactions on Communications, vol. 65, no. 2, pp. 728-739, Feb. 2017.

[23] D. Lopez-Perez, A. Valcarce, G. de la Roche, and J. Zhang, "OFDMA femtocells: a roadmap on interference avoidance", IEEE Communications Magazine, vol. 47, no. 9, pp. 41-48, Sept. 2009.

[24] A. R. Elsherif, W. P. Chen, A. Ito, and Z. Ding, "Adaptive resource allocation for interference management in small cell networks", IEEE Transactions on Communications, vol. 63, no. 6, pp. 2107-2125, Jun. 2015.

[25] A. Bhatia and R. C. Hansdah, "RD-TDMA: a randomized distributed TDMA scheduling for correlated contention in WSNs", 2014 28th International Conference on Advanced Information Networking and Applications Workshops, Victoria, BC, 2014, pp. 378-384.

[26] Z. Zhang, L. Song, Z. Han, and W. Saad, "Coalitional games with overlapping coalitions for interference management in small cell networks," IEEE Transactions on Wireless Communications, vol. 13, no. 5, pp. 2659-2669, May. 2014.

[27] Y. Dong, M. Sheng, S. Zhang, and C. Yang, "Coalition based interference mitigation in femtocell networks with multi-resource allocation," 2014 IEEE International Conference on Communications (ICC), Sydney, NSW, 2014, pp. 2695-2700.

[28] W. Shin, W. Noh, K. Jang, and H. H. Choi, "Hierarchical interference alignment for downlink eterogeneous networks", IEEE Transactions on Wireless Communications, vol. 11, no. 12, pp. 4549-4559, Dec. 2012.

[29] Y. Xu and S. Mao, "Stackelberg game for cognitive radio networks with MIMO and distributed interference alignment", IEEE Transactions on Vehicular Technology, vol. 63, no. 2, pp. 879-892, Feb. 2014.

[30] Q. Wang, J. Wang, and Y. Guan, "Price-Based unit commitment with wind power utilization constraints", IEEE Transactions on Power Systems, vol. 28, no. 3, pp. 2718-2726, Aug. 2013.

[31] M. Wang, H. Tian, and G. Nie, "Energy efficient power and subchannel allocation in dense OFDMA small cell networks", 2014 IEEE 80th Vehicular Technology Conference (VTC2014-Fall), Vancouver, BC, 2014, pp. 1-5.

[32] W. Yu, T. Kwon, and C. Shin, "Multicell Coordination via Joint Scheduling, Beamforming, and Power Spectrum Adaptation", IEEE Transactions on Wireless Communications, vol. 12, no. 7, pp. 1-14, Jul 2013.

[33] J. Xiao, C. Yang, J. Wang, and H. Dai, "Joint interference management in ultra-dense small cell networks: A multi-dimensional coordination," 2016 8th International Conference on Wireless Communications \& Signal Processing (WCSP), Yangzhou, 2016, pp. 1-5.

[34] L. Ruan, V. Lau, and M. Z. Win, "The feasibility conditions for interference alignment in MIMO networks", IEEE Transactions on Signal Process, vol. 61, no. 8, pp. 2066-2077, Apr. 2013.

[35] K. Gomadam, V. R. Cadambe, and S. A. Jafar, "A distributed numerical approach to interference alignment and applications to wireless interference networks," IEEE Transactions on Information Theory, vol. 57, no. 6, pp. 3309-3322, Jun. 2011.

[36] R. Tresch, M. Guillaud, and E. Riegler, "On the achievability of interference alignment in the K-user constant MIMO interference channel," 2009 IEEE/SP 15th Workshop on Statistical Signal Processing, Cardiff, 2009 , pp. 277-280.

[37] R. S. Cheng and S. Verdu, "Gaussian multiaccess channels with ISI: capacity region and multiuser water-filling", IEEE Transactions on Information Theory, vol. 39, no. 3, pp. 773-785, May. 1993.

[38] 3GPP, "TR 36.814 evolved universal terrestrial radio access (E-UTRA) further advancements for E-UTRA physical layer aspects," 2010. 


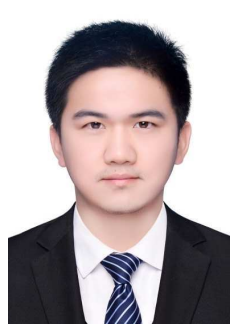

Jia Xiao received his Bachelor Degree degree in Communication Engineering at Xidian University in 2015. He is currently pursuing his Master Degree at Xidian University since 2015. He works in the GUIDE research team, which is guided by Dr. Chungang Yang. His research interests include social-aware D2D communications, self-organizing ultra-dense small cell networks, and overlapping coalitional games.

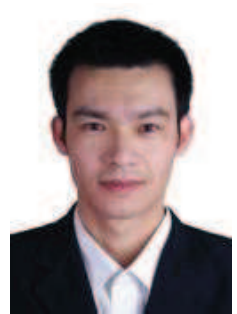

Chungang Yang ( $\left.\mathrm{S}^{\prime} 09, \mathrm{M}^{\prime} 12\right)$ is currently an associate professor at Xidian University, where Dr. Yang leads the research team of "GUIDE, Game, Utility, Intelligent computing Design for Emerging communications". Dr. Yang received his Bachelor and Doctoral Degree at Xidian University, Xi'an, China, in 2006 and 2011, respectively. Between Sep. 2010 and March 2011, he held the visiting scholar position in the department of electrical and computer engineering at Michigan Technological University. Between March 2015 and March 2016, he held the visiting scholar position in the department of electrical and computer engineering at University of Houston. His research interests include resource and interference management, network optimization, and mechanism design for cognitive radio networks, heterogeneous cellular networks, and game theory for wireless communication and computing networks.

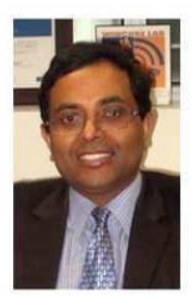

Alagan Anpalagan received the B.A.Sc. M.A.Sc. and $\mathrm{Ph} . \mathrm{D}$. degrees in Electrical Engineering from the University of Toronto, Canada. He joined the Department of Electrical and Computer Engineering at Ryerson University in 2001 and was promoted to Full Professor in 2010. He served the department as Graduate Program Director (2004-09) and the Interim Electrical Engineering Program Director (2009-10). During his sabbatical (2010-11), he was a Visiting Professor at Asian Institute of Technology and Visiting Researcher at Kyoto University. Dr. Anpalagan's industrial experience includes working at Bell Mobility, Nortel Networks and IBM Canada. Dr. Anpalagan directs a research group working on radio resource management (RRM) and radio access \& networking (RAN) areas within the WINCORE Lab. His current research interests include cognitive radio resource allocation and management, wireless cross layer design and optimization, cooperative communication, M2M communication, small cell networks, energy harvesting and green communications technologies. He is a Senior Member of the IEEE.

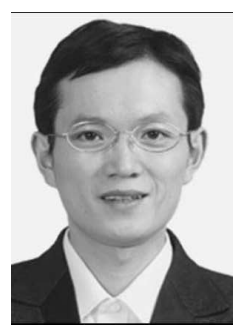

Qiang Ni (M'04-SM'08) received his Ph.D. degree in Engineering from Huazhong University of Science and Technology, Wuhan, in 1999. He is currently a Full Professor and the Head of Communication Systems Research Group at the School of Computing and Communications, Lancaster University, Lancaster, UK. His research interests include future generation communications and networking systems, energy and spectrum efficient green wireless communications, 5G, SDN, game theory, heterogeneous networks, cognitive radio network systems, ultradense networks, cloud networks, energy harvesting, IoTs, vehicular networks, big data analytics, etc. He had published more than 180 research papers in international journals and conferences. He was an IEEE 802.11 Wireless Standard Working Group Voting Member and a contributor to various IEEE Wireless Standards.

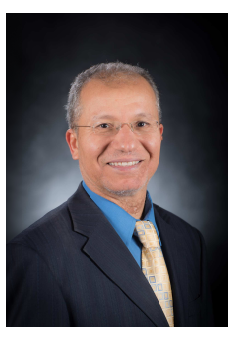

Mohsen Guizani (S'85-M'89-SM'99-F'09) received the B.S. (with distinction) and M.S. degrees in electrical engineering, the M.S. and Ph.D. degrees in computer engineering from Syracuse University, Syracuse, NY, USA, in 1984, 1986, 1987, and 1990, respectively. He is currently a Professor and the ECE Department Chair at the University of Idaho, USA. Previously, he served as the Associate Vice President of Graduate Studies, Qatar University, Chair of the Computer Science Department, Western Michigan University, and Chair of the Computer Science Department, University of West Florida. He also served in academic positions at the University of Missouri-Kansas City, University of ColoradoBoulder, Syracuse University, and Kuwait University. His research interests include wireless communications and mobile computing, computer networks, mobile cloud computing, security, and smart grid. He currently the Editorin-Chief of the IEEE Network, serves on the editorial boards of several international technical journals and the Founder and the Editor-in-Chief of Wireless Communications and Mobile Computing journal (Wiley). He is the author of nine books and more than 500 publications in refereed journals and conferences. He guest edited a number of special issues in IEEE journals and magazines. He also served as a member, Chair, and General Chair of a number of international conferences. He received the teaching award multiple times from different institutions as well as the best Research Award from three institutions. He received the 2017 IEEE ComSoc Recognition Award for his contribution to Wireless Communications. He was the Chair of the IEEE Communications Society Wireless Technical Committee and the Chair of the TAOS Technical Committee. He served as the IEEE Computer Society Distinguished Speaker from 2003 to 2005. He is a Fellow of IEEE and a Senior Member of ACM. 